Research Article

\title{
Exploitation of Indigenous Wine Yeasts from Spontaneously Fermenting Grape must and Vineyard Soil in Beijing, China
}

\author{
Qianwen Zhang ${ }^{1}$, Hui Li ${ }^{2}$, Xiangyu Sun ${ }^{1}$, Weidong Huang ${ }^{1}$, Jicheng Zhan ${ }^{1, *}$ \\ ${ }^{1}$ College of Food Science and Nutritional Engineering, Beijing Key Laboratory of Viticulture and Enology, China Agricultural University, Beijing 100083, China \\ ${ }^{2}$ Food Quality \& Safety Center, Nutrition \& Health Research Institute, COFCO Corporation, Beijing 102209, China
}

\section{ARTICLE INFO}

\section{Article History}

Received 06 April 2020

Accepted 29 July 2020

Keywords

Wine yeast

vineyard soil

spontaneous fermentation

5.8S ITS

microbial ecology

\begin{abstract}
The investigation of indigenous wine yeast can facilitate the production of distinctive Beijing wine by providing wineries with more choices in distinctive wine yeasts. Wine yeasts that were isolated from grape must during spontaneous fermentation and from vineyard soil were preliminarily classified by their colony morphologies when cultured on Wallerstein Laboratory Nutrient (WLN) medium and then were identified by 5.8S ITS sequence analysis. Four selected strains were involved in inoculated fermentation tests. The results showed that five yeast species were isolated from the grape must, and five non-Saccharomyces yeast species were found in vineyard soil. The colony morphology of Pichia occidentalis on the WLN medium was described herein for the first time. An effective way which could be used for isolate and select wine yeasts from soil samples on WLN medium was described. And one of the selected indigenous strains shared over $99 \%$ similarity on $5.8 \mathrm{~S}$ ITS sequence with commercial wine yeast, which indicated that commercial yeast might have influenced the local yeast diversity. The two selected indigenous Saccharomyces cerevisiae strains, which showed good fermentation performances, could be applied in the local wine industry in the future.
\end{abstract}

\section{GRAPHICAL ABSTRACT}
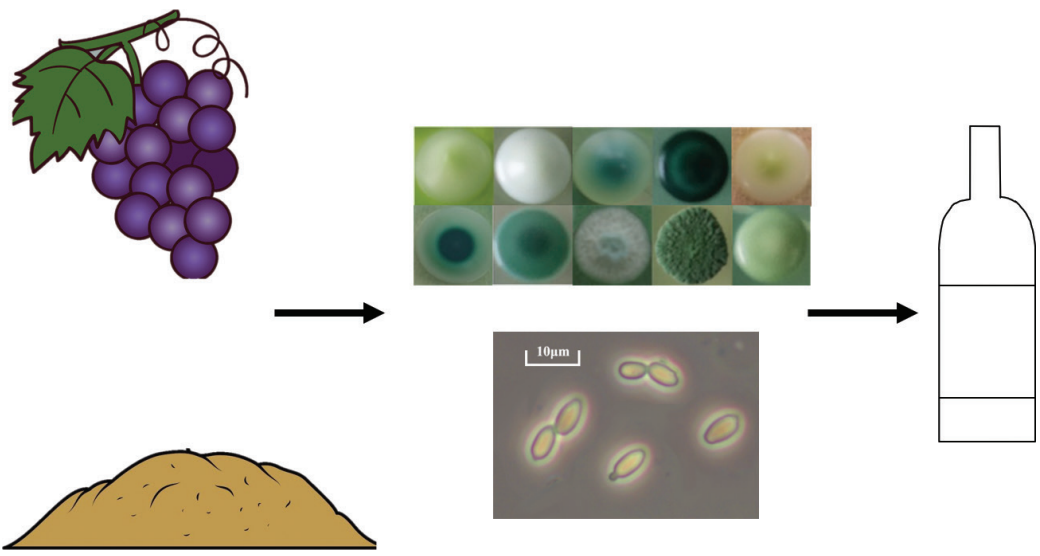

(c) 2020 International Association of Dietetic Nutrition and Safety. Publishing services by Atlantis Press International B.V. This is an open access article distributed under the CC BY-NC 4.0 license (http://creativecommons.org/licenses/by-nc/4.0/).

\section{INTRODUCTION}

As vast majority of wine demand being met by importation, China became the fourth largest wine importer worldwide after Germany, the UK and the US [1]. With fast development of Chinese wine industry, the need for indigenous wine production in China has quickly increased. Beijing, which has long history of wine production, has become one of the biggest wine-consuming cities

Corresponding author. Email: zhanjicheng@cau.edu.cn

Peer review under responsibility of the International Association of Dietetic Nutrition and Safety in China. However, Beijing has not yet formed its own style of wine. One important reason for that is the use in the wineries of similar, imported commercial yeasts, usually a single strain of Saccharomyces cerevisiae.

Wine yeasts have significant effects on the quality of wine. Different species of yeasts produce different metabolites during fermentation, which determine the chemical composition of the wine. The selection of wine yeasts that are right for different type of region and microclimate is becoming even more important [2-5]. Generally, there are two methods for starting fermentation: inoculation with commercial active dry yeasts and spontaneous fermentation. 
The commercial starters have the advantages of high fermentation abilities, producing predictable aroma compositions without toxic metabolites and resulting in a wine of consistent quality [6]. However, commercial strains of $S$. cerevisiae are often similar strains without typical characteristics [7]. Moreover, commercial active dry yeasts occasionally fail to be the dominant microorganism during the fermentation and they might be replaced by indigenous yeast. These starters may be replaced by indigenous wine yeasts that are more suitable for local wine grapes [8-10]. Spontaneous fermentation is usually tried by small wineries who expect to make special wines [6]. The spontaneous fermentation of wine is an extremely complex process, characterized by a high microbial diversity [11]. Thus, to select indigenous yeast from spontaneous fermentation can be challengeable. Wallerstein Laboratory Nutrient (WLN) medium was developed for selective isolation of yeasts and bacteria from breweries and industrial fermentation environments [12]. It was widely used in the isolation of microorganisms from fermentation because different species show different colony morphology characteristics on WLN medium $[8,9,13]$.

In recent years, many studies have been conducted to investigate indigenous wine yeasts around the world, including in France, Australia, Spain, Italy, Hungary, the United States, etc. [9,10,14-18]. In China, researchers also have investigated indigenous wine yeasts. However, most of these studies are focused on northwestern China, including Sinkiang, Ningxia, Gansu and Shaanxi, four provinces that have produced wines that have received international awards in recent years [19]. In order to produce distinctive wine and meet the demand of large scale wineries in Beijing, indigenous wine yeasts should be studied.

To identify the diversity of indigenous wine yeasts, investigating yeast ecology during spontaneous fermentation is an effective way, especially for S. cerevisiae [20,21-24]. However, since grape must is only available in autumn, there is only one season in a year to investigate the spontaneous fermentation. In other seasons, however, especially in winter, the soil in the vineyard can be studied [23]. Besides, collecting samples from different material sources may provide more chances for isolating various species of wine yeasts in the same place.

In this study, wine yeasts were isolated from Cabernet Sauvignon grape must during the spontaneous fermentation and from the vineyard soils of Cabernet Sauvignon, Chardonnay and Sauvignon Blanc. The fermentation tests of selected S. cerevisiae and Starmerella bacillaris proved their potential in wine-making in the future. In order to take full advantage of the WLN medium and simplify the identification of wine yeasts, an attempt to analyze the relationship between yeasts' colony morphologies on WLN medium and their genotypes was also made. To our knowledge, this work is the first study using WLN medium to isolate wine yeasts from soil samples, which provides an effective way to investigate wine yeasts when there were no grape berries. The results reported represent the first step needed to exploit the useful indigenous wine yeasts in Beijing wine region.

\section{MATERIALS AND METHODS}

\subsection{Reference Commercial Strains}

Two commercial active dry yeasts, which were developed especially for red wine and used in Chateau Lion, served as the control strains. S. cerevisiae RB2 and S. cerevisiae F15 from Laffort Oenologie (Bordeaux, France).

Strains were activated in yeast extract peptone dextrose (YPD) broth ( $1 \%$ yeast extract, $2 \%$ peptone, and $2 \%$ glucose) at $28^{\circ} \mathrm{C}$ for $12 \mathrm{~h}$, and then a purification by sequential single colony isolation was conducted on WLN agar (Nissui Bio-technologies Co., Ltd., Qingdao, China).

\subsection{Grape Berry Sampling and Fermentation}

Cabernet Sauvignon grapes were cultivated in the vineyard of Chateau Lion $\left(39^{\circ} 47^{\prime} 32.40^{\prime \prime}+116^{\circ} 13^{\prime} 14.91^{\prime \prime}\right)$. The mature grape clusters were manually picked during the 2014 vintage. They were then subjected to a laboratory-scale spontaneous fermentation test. In total, $500 \mathrm{~mL}$ grape must (sugar content $23^{\circ} \mathrm{Brix}$ ) were collected in each $1 \mathrm{~L}$ sterile conical flask for spontaneous fermentations in triplicate (M1, M2, and M3). Aliquots of $0.1 \mathrm{~mL}$ initial grape must were immediately spread on the WLN agar to isolate microbes before fermentation. The WLN agar was incubated at $28^{\circ} \mathrm{C}$ for 5 days. Subsequently, $36 \mathrm{mg} / \mathrm{L} \mathrm{SO}_{2}$ was added to the must. The conical flasks were incubated at $28^{\circ} \mathrm{C}$. The ethanol content was detected daily by the High Performance Liquid Chromatography (HPLC) method, following the protocol reported previously [25].

\subsection{Soil Sampling and Yeast Enrichment}

The three soil samples came from the vineyards of Cabernet Sauvignon, Chardonnay and Sauvignon Blanc, respectively, in Chateau Changyu AFIP Global (402'․ $\left.32^{\prime \prime}+116^{\circ} 54^{\prime} 24.26^{\prime \prime}\right)$. During the winter, when the grapes were buried underground, soil samples were collected by sterile tools. Each sample included soils and deadwood of the grapes.

Two methods of yeast enrichment were adopted according to the results of a preliminary experiment. In the first method, sterile $250 \mathrm{~mL}$ conical flasks were filled with $2 \mathrm{~g}$ soil and $150 \mathrm{~mL}$ YPD broth with the addition of $60 \mathrm{mg} / \mathrm{L} \mathrm{SO}_{2}, 50 \mu \mathrm{g} / \mathrm{mL}$ chloramphenicol and $10 \%(\mathrm{v} / \mathrm{v})$ ethanol. In the other method, $2 \mathrm{~g}$ soil was added into the same liquid YPD medium with same additions mentioned above except for ethanol. All conical flasks were then sealed with a sterile sealing film and incubated for one week at $28^{\circ} \mathrm{C}, 150 \mathrm{rpm}$.

Preliminary experiments showed that the first method mentioned above provided dependable isolation of wine yeasts that were tolerant to ethanol, especially for Saccharomyces, and controlled filamentous fungi (mold) growth, whereas the second method was used to isolate wine yeasts that were not tolerant to ethanol.

\subsection{Isolation of Wine Yeast}

One-milliliter samples of grape must were taken every day from the 3rd day to the 10th day of fermentation. For the soil enrichment culture, $1 \mathrm{~mL}$ samples were taken from the 7 th day of the enrichment culture. Serial decimal dilutions were made in sterile Milli-Q water, and aliquots $(0.2 \mathrm{~mL}$ each) were spread on WLN agar plate and incubated at $28^{\circ} \mathrm{C}$ for 5 days. 
Colonies were differentiated and grouped by their morphologies and color characteristics, as described by Cavazza et al. [26] and Pallmann et al. [13]. Colonies were grouped into seven types to make successive identifications. Four groups had similar shapes and textures. They were both round, with smooth, creamy, and buttery surfaces. They were named according to color as White (W), Pale green (P), Green (G), and Dark green (D). The other three groups had irregular shapes or distinctive textures and were named Flour (F), Radial (R), and Bulge (B). Each colony picked out from the grape must was named according to their source (M1, M2, and M3), morphology group (W, P, G, D, F, R, and B), and the day they were picked out during fermentation (3-10). Furthermore, the colonies isolated from the soil samples were named according to the grape varieties and a serial number. From each morphology type from different grape must and fermentation stages, representative strains were randomly selected. All selected strains were purified by sequential single colony isolation to avoid other microorganism contamination.

During the spontaneous fermentation, the serially diluted plates with total colony numbers of 30-300 were picked out to predict the yeast populations, the microscopic features were observed and recorded during the selection and purification stage. Micrographs were taken by phase contrast microscope (Axio Scope A1, Carl Zeiss Co., Ltd., Göttingen, Germany). In total, 63 isolates were selected for subsequent analysis and stored in the YPD agar slope (1\% yeast extract, $2 \%$ peptone, $2 \%$ glucose, and $2 \%$ agar) at $4^{\circ} \mathrm{C}$. The graphs of yeast ecology during spontaneous fermentation were made by software Origin (version 8.5; OriginLab Corporation, Northampton, MA, USA).

\section{5. $5.8 \mathrm{~S}$ ITS Sequence Analysis of Selected Yeasts}

Genomic DNA of yeast was extracted by the method of TIANamp Yeast DNA Kit (Tian Gen Co., Ltd., Beijing, China). The species of the selected yeasts were identified by the 5.8S ITS rDNA gene region. PCR with primers ITS1 (5'-TCCGTAGGTGAACCTGCGG-3') and ITS4 (5'-TCCTCCGCTTATTGATATGC-3') was amplified in a BIO$\mathrm{RAD} \mathrm{T}_{100}{ }^{\mathrm{Tm}}$ Thermal Cycler (Bio-Rad Laboratories, Inc., Hercules, CA, USA) programmed as described in Guillamón et al. [27]. Aliquots of $6 \mu \mathrm{L}$ of amplified product were subjected to $1 \%(\mathrm{w} / \mathrm{v})$ agarose gel electrophoresis at $100 \mathrm{~V}$ constant voltages for $30 \mathrm{~min}$. The gels were stained with ethidium bromide and photographed using a Gel Doc XR system (Bio-Rad Laboratories, Inc.). In total, 66 amplified products (including three commercial yeast strains) with positive results were sent to BGI TechSolutions Co., Ltd. (Beijing, China) for sequencing. Sequence analysis was performed by the basic local alignment search tool (Blast) in the National Center for Biotechnology Information database. The dendrogram was constructed with the Mega 6.0 software (Pennsylvania State University, USA) [28].

\subsection{Inoculated Fermentation Tests}

Two S. cerevisiae strains, M1W10 and M2W10, two S. bacillaris strains, M1P9 and M3P9 were used in inoculated fermentation test. The commercial wine yeast F15 was used as control yeast strain in the fermentation test.
The five yeast strains were pre-cultured in YPD broth to log-phase. The initial yeast inoculums of $10^{6} \mathrm{CFU} / \mathrm{mL}$ were inoculated to $800 \mathrm{~mL}$ grape must (sugar content $23^{\circ}$ Brix) with $36 \mathrm{mg} / \mathrm{L} \mathrm{SO}_{2}$. Fermentations were conducted in $1 \mathrm{~L}$ glass bottles, which were incubated at $22^{\circ} \mathrm{C}$. The ethanol content was detected daily by the HPLC method. Fermentation assay for each yeast strains was performed in triplicate.

The yeasts' fermentation performances were analyzed by the glucose, fructose, glycerol, ethanol, succinic acid, lactic acid, and acetic acid contents in the experimental wine. These contents in grape juice and wine were determined by HPLC method in triplicate, following the protocol reported previously [25]. Sensory test was conducted to evaluate the appearance (10 points), aroma (30 points), taste (40 points) and overall quality ( 20 points) of wine. According to the evaluation criteria described in GB/T 15038-2006 [29], 11 wine connoisseurs were invited to a blind tasting test of the five groups of wines. The score sheet was designed according to Ronald [30] with minor modifications. The results were analyzed by Microsoft Excel 2010.

\section{RESULTS}

\subsection{Isolation of Wine Yeasts}

During the spontaneous fermentation of three groups of Cabernet Sauvignon grape must (M1, M2, and M3), more than 2000 colonies were isolated. Fifty-three representative colonies were selected for 5.8S ITS sequence analysis. On the other hand, over 100 colonies were isolated from the soil samples. Among these colonies, 10 were selected to undergo molecular identification.

From the grape must, the five species identified were $S$. cerevisiae, Hanseniaspora uvarum, Hanseniaspora vineae, S. bacillaris, and Pichia occidentalis. While from the vineyard soil samples, the five species found were Pichia kluyveri, H. uvarum, Schizosaccharomyces pombe, Pichia fermentans, and Torulaspora delbrueckii. No Saccharomyces were isolated from the soil samples. Since two enrichment methods used, the vineyard distribution of different species were illustrated in enrichment cultures without ethanol and enrichment cultures with ethanol, respectively (Table 1 ).

Table 1 Colony numbers of different yeasts distributed among vineyard soil samples

\begin{tabular}{|c|c|c|c|c|c|c|}
\hline \multirow{3}{*}{$\begin{array}{l}\text { Yeast } \\
\text { species }\end{array}$} & \multicolumn{6}{|c|}{ Soil samples } \\
\hline & \multicolumn{2}{|c|}{$\begin{array}{l}\text { Cabernet } \\
\text { Sauvignon }\end{array}$} & \multicolumn{2}{|c|}{ Chardonnay } & \multicolumn{2}{|c|}{ Sauvignon Blanc } \\
\hline & $\mathrm{CnE}^{*}$ & $\mathrm{CE}^{* *}$ & $\mathrm{CnE}$ & CE & $\mathrm{CnE}$ & $\mathrm{CE}$ \\
\hline P. kluyveri & - & 5 & - & 12 & - & 6 \\
\hline H. uvarum & - & - & 4 & - & 2 & - \\
\hline S.pombe & - & - & 2 & 18 & 5 & 8 \\
\hline P. fermentans & 2 & - & - & - & - & - \\
\hline T. delbrueckii & - & 5 & - & 3 & 3 & 6 \\
\hline
\end{tabular}

"CnE indicates enrichment cultures without ethanol. " ${ }^{*} \mathrm{CE}$ indicates enrichment cultures with ethanol. -: not detected. 


\section{Saccharomyces cerevisiae}

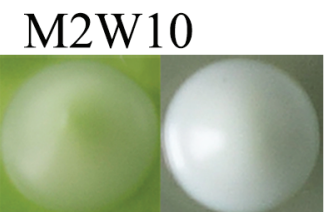

common purified typical purified

\section{Hanseniaspora uvarum}

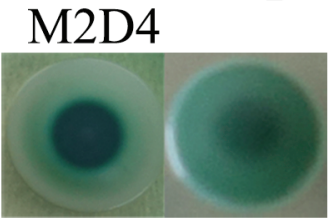

M3D4

common purified typical purified

\section{Hanseniaspora vineae}
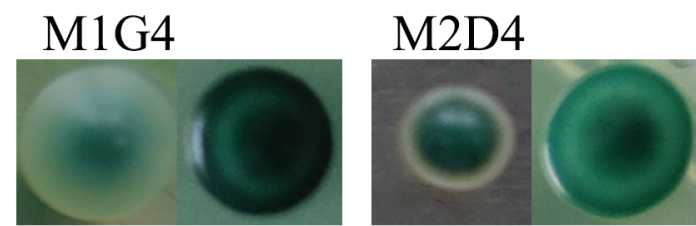

common purified typical purified

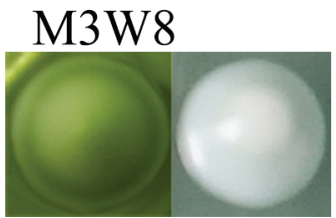

typical purified

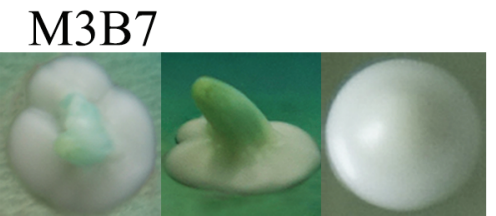

typical typical* purified
M1B5

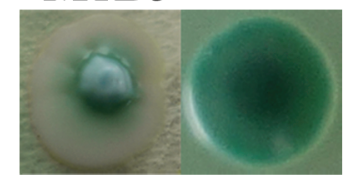

typical purified

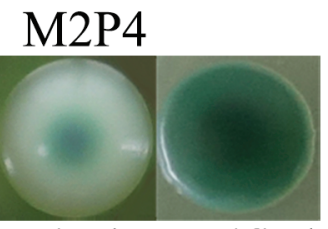

typical purified

\section{Starmerella bacillaris}

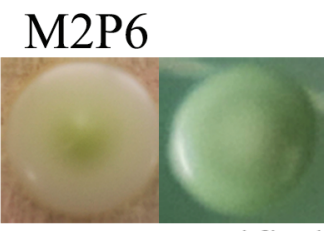

M2R6

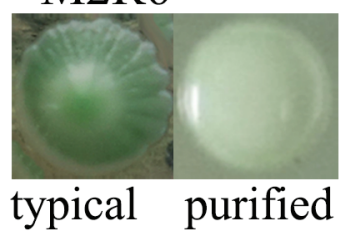

\section{Pichia occidentalis}

M3F5

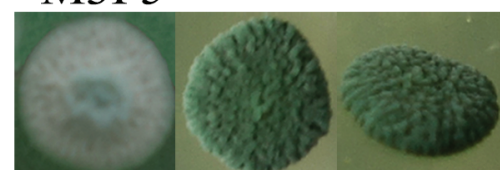

common purified purified*

Figure 1 Different colony morphology types on the WLN medium of five wine yeast species. Common: The most common colony morphology type on the WLN medium of each species during the selection stage. Typical: A typical colony morphology type on the Wallerstein Laboratory Nutrient (WLN) medium different from common colonies during the isolation stage, usually only one colony of such type was found. Purified: Colony morphology type on the WLN medium after purification of corresponding strain. *: Side view of the colony.

\subsection{Different Morphology Types of Specific Species}

According to 5.8S ITS sequence analysis and corresponding colony photos, there were four species that had more than one colony morphology type on the WLN medium. These species are S. cerevisiae, $H$. uvarum, $H$. vineae, and $S$. bacillaris. The most common colony and some typical colonies of these species on the WLN plate are illustrated in Figure 1. Because some species have different colony morphologies among selection and purification stages, their colony photos when they were selected before purification were compared with the corresponding colony photos after purification (Figure 1). The names of these strains follow the naming scheme mentioned in methods. Strains from same species showed similar colony characteristics after purification, although some small differences still could be found. Those small differences in colony morphology after purification were related to the characteristics on the colonies the first time when they were isolated. For example, S. cerevisiae M2W8 showed a more greenish hue on the top of the colony after purification, whereas the purified colony of S. cerevisiae M3W8 showed a slight green periphery. These characteristics are related to M2W8, M3W8 typical colony morphology, respectively (Figure 1).

The $P$. occidentalis (M3F5) on the WLN plate showed white to dark green colonies, with a wrinkled and floury surface (Figure 1). The micrograph of $P$. occidentalis is shown in Figure 2, and the species exhibits elongated ellipsoidal cells and multilateral budding reproduction.

\subsection{Yeast Ecology during the Spontaneous Fermentation}

The initial microbe proportion in the grape must before fermentation was $100 \%$ H. vineae and $H$. uvarum. Because it was hard 
to distinguish the two Hanseniaspora species on the WLN agar medium, in Figure 3, the colony numbers of the two species were shown as a sum of the "Hanseniaspora sp." colony number. However, the randomly selected Hanseniaspora species strains identified by sequence analysis showed that the $H$. vineae dominates over $H$. uvarum on the 3 rd day of fermentation; while the $H$. uvarum became the dominant species from the 4 th day to the 5 th day in M1, M2, and M3. To explore dominant strains in each stage of spontaneous fermentation, populations of different yeast species at the 3rd day to the 10th day in the grape must of spontaneous fermentation (M1, M2, and M3) are shown in Figure 3. The ethanol content was shown by line to indicate the fermentation progress. At the end of the fermentation, the ethanol contents of M1, M2, and M3 were $13.96 \%, 13.25 \%, 13.46 \%$ (v/v), respectively. The $P$. occidentalis only showed on the 5th day of fermentation in M1. In particular, the colony number of M1 was smaller than that of M2 and M3. On the 3rd day, an additional $18 \mathrm{mg} / \mathrm{L} \mathrm{SO}_{2}$ was added into the M1 must to inhibit mold growth. The higher concentrate of sulfur dioxide may also inhibit the population of wine yeasts in M1 [31].

\subsection{Genotypes of the S. cerevisiae Strains}

To learn whether the selected $S$. cerevisiae strains come from the commercial yeasts or not, the genotypes of the selected $S$. cerevisiae

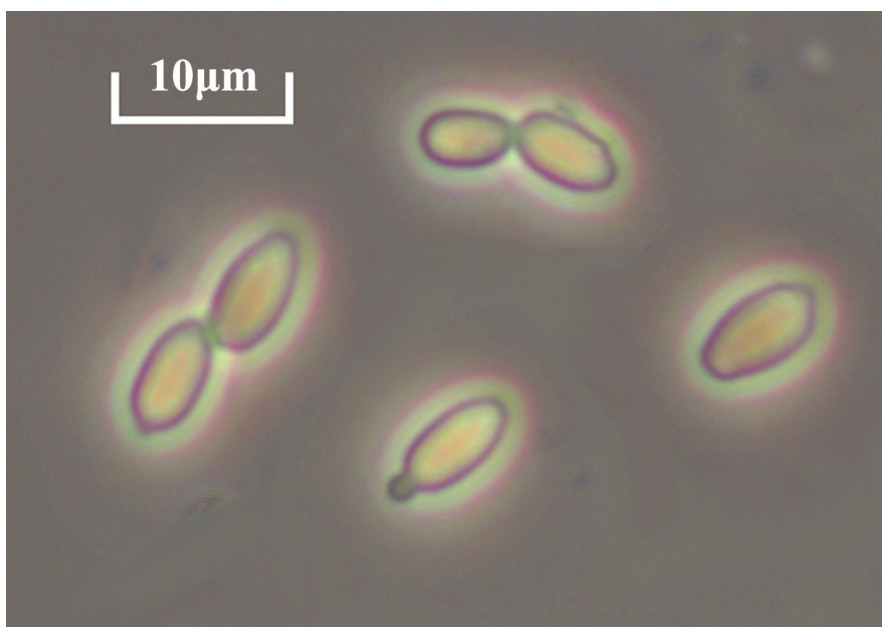

Figure 2 Microscopic morphology of Pichia occidentalis in the logarithmic phase. strains and the commercial strains used in the winery were compared. However, there were two different strains isolated from the commercial yeast RB2. These were renamed as R1 and R2. A phylogenetic dendrogram (Figure 4) based on the 5.8S ITS sequences of the $S$. cerevisiae strains was constructed to analyze the homology between selected strains and commercial strains. The Neighborjoining tree was constructed based on the Kimura 2-parameter model, using the Bootstrap method in phylogeny test, with 1000 bootstrap replications. Strains were grouped into I, II, III, and other independent strains, judged by bootstrap values that were over $70 \%$. The colony morphology (after purification) of each S. cerevisiae strains is shown next to the dendrogram.

\subsection{Analysis of Experimental Wine Inoculated with Selected Wine Yeasts}

Saccharomyces cerevisiae strains M1W10 and M2W10 dominated at the end stage of spontaneous fermentation, with rather distant phylogenetic relationships to commercial yeasts (Figure 4), were selected to test for their fermentation abilities. Except for $S$. cerevisiae, $S$. bacillaris was dominant yeast especially during the middle stage (6-8 days) of fermentation. And S. bacillaris was one of the wine yeasts which existed in the end stage of fermentation. On the 9th day of the fermentation, the alcohol content was close to $14 \%$ $(\mathrm{v} / \mathrm{v})$, a high concentration that can select wine yeasts which were tolerant to alcohol. Moreover, S. bacillaris strains that were isolated from grape and wine have been reported by Chinese researchers for many times in recent years [32-34,19]. These strains have been found in seven cities that belong to four wine regions around China, its fermentation ability deserved to be elucidated. Therefore, S. bacillaris strains M1P9 and M3P9, which were isolated from the 9th day of the fermentation, were also used in the fermentation tests.

The three bottles of grape must inoculated with selected $S$. cerevisiae strains fermented rapidly. The fermentation finished on the 7 th day. However, grape musts inoculated with $S$. bacillaris fermented very slowly. Until the 15 th day of fermentation, there were no more alcohol produced in the grape must; furthermore, high sugar contents remained in the grape must. This means the fermentation of grape must inoculated with $S$. bacillaris was over before the sugars were used up in the process. The contents of sugars and acids of these experimental wines were shown in Figure 5. In addition, in the initial grape must, the content of glucose, fructose, succinic acid, lactic acid, acetic acid, glycerol, and ethanol were 95.56,
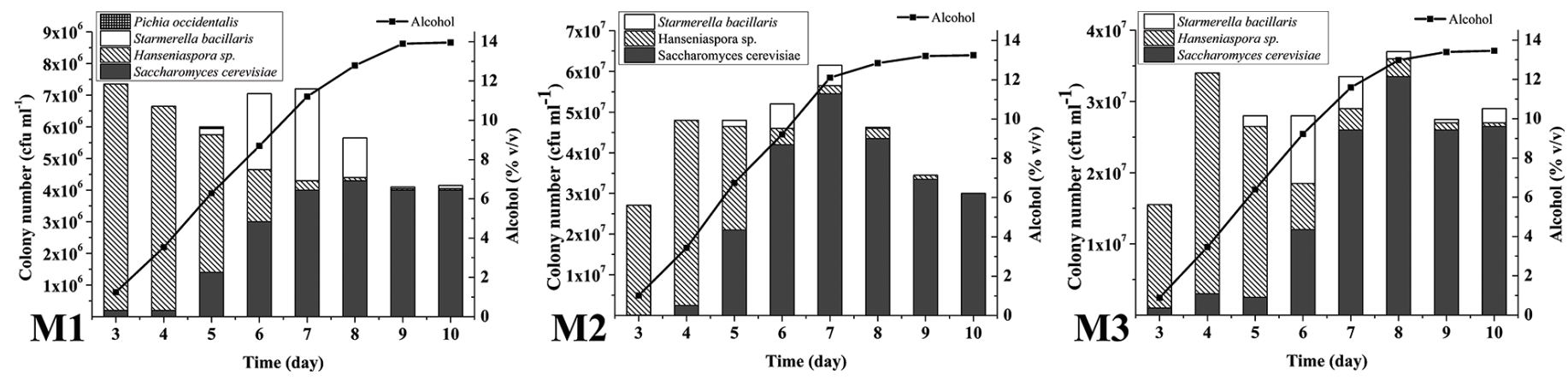

Figure 3 The yeast ecology kinetics during the spontaneous fermentation of M1, M2, and M3. The colony numbers are expressed as CFU/mL, and the contents of ethanol are expressed as \% (v/v). 


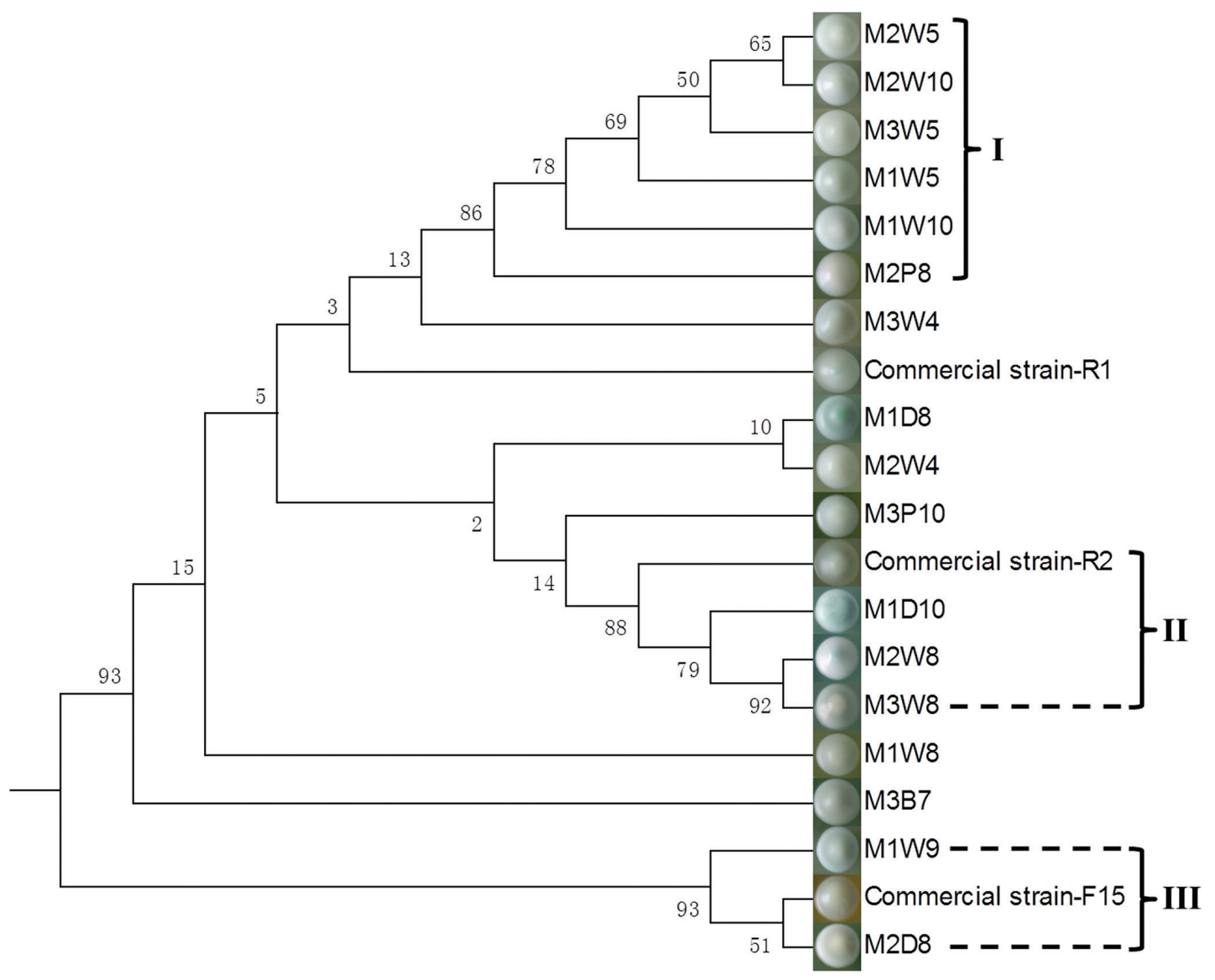

Figure $4 \mid$ Dendrogram obtained by cluster analysis of the 5.8S ITS sequence of Saccharomyces cerevisiae strains.
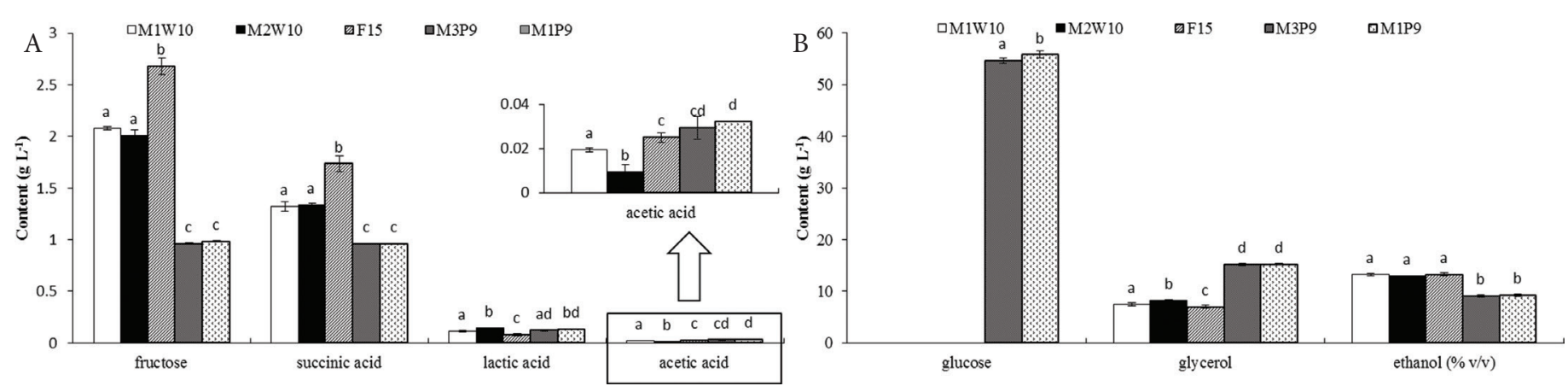

Figure 5 (A) Contents of fructose, succinic acid, lactic acid, and acetic acid in five experimental wines; (B) Contents of glucose, glycerol, and ethanol in five experimental wines. All values are expressed as $\mathrm{g} / \mathrm{L}$, with the exception of the ethanol contents, which are expressed as $\%$ (v/v). Different letters indicate the statistically significant differences (Duncan's multiple range test, $p<0.05$ ) among different time point. Values are mean \pm SD.

$121.63,0.09,0,0,1.02 \mathrm{~g} / \mathrm{L}$, and $0.11 \%(\mathrm{v} / \mathrm{v})$, respectively. The results of sensory tests were shown in Table 2.

\section{DISCUSSION}

Some findings were obtained by using the WLN medium to isolate the wine yeast. First, as seen in Figures 1 and 4, some typical colony characteristics among the same species may reveal some genetic differences among strains. However, in our study, 98\% of the colonies on the WLN medium had common colony morphologies as mentioned in previous studies $[13,26]$. Therefore, the WLN medium is a good way to differentiate yeast species preliminarily. To obtain accurate results, several of the most common colonies belong to each morphology type, and all typical colonies should be picked out to enable further molecular identifications. Second, although most relevant studies focused on the use of WLN medium to isolate wine yeasts present on the surfaces of the grapes or in fermenting must $[8,9,33]$, in this study, the WLN medium was also used to isolate wine yeasts from vineyard soil samples. Five non-Saccharomyces species of wine yeasts were isolated as shown in Table 1. Among the five yeast species, H. uvarum and T. delbrueckii were frequently found in wine fermentations $[35,36]$. Non-Saccharomyces yeasts have been playing more and more 
Table 2 Descriptive analysis and scores in the sensory evaluation of wines fermented by five strains of selected yeasts

\begin{tabular}{|c|c|c|c|c|c|}
\hline & Appearance & Aroma & Taste & Overall quality & Total score \\
\hline M1W10 & Brilliant (19.88 \pm 0.35$)$ & $\begin{array}{l}\text { Rich aroma, violet, cherry, a little } \\
\text { grassy }(26.50 \pm 1.77)\end{array}$ & $\begin{array}{l}\text { A little sharp acidity, a little slight } \\
\quad(34.88 \pm 3.23)\end{array}$ & Good $(8.50 \pm 0.76)$ & $89.75 \pm 5.01$ \\
\hline M2W10 & Brilliant $(19.88 \pm 0.35)$ & $\begin{array}{l}\text { Rich aroma, cherry, pear, rose, violet } \\
(27.25 \pm 1.91)\end{array}$ & $\begin{array}{l}\text { Full, balanced, a long, fruity finish } \\
(35.50 \pm 2.27)\end{array}$ & Good $(9.00 \pm 0.53)$ & $91.13 \pm 4.32$ \\
\hline F15 & Brilliant (19.88 \pm 0.35$)$ & $\begin{array}{l}\text { Rich aroma, pear, a little grassy } \\
(25.75 \pm 0.89)\end{array}$ & $\begin{array}{l}\text { Sharp acidity, a prolonged flavour } \\
\text { in mouth }(33.00 \pm 3.21)\end{array}$ & Superior $(7.81 \pm 0.65)$ & $86.44 \pm 3.66$ \\
\hline M3P9 & Brilliant (19.88 \pm 0.35$)$ & $\begin{array}{l}\text { Standard presence of a varietal, } \\
\text { strawberry }(25.75 \pm 1.39)\end{array}$ & $\begin{array}{l}\text { Slight flavour, balanced } \\
\quad(33.38 \pm 3.78)\end{array}$ & Superior $(7.75 \pm 0.71)$ & $86.75 \pm 5.15$ \\
\hline M1P9 & Brilliant (19.88 \pm 0.35$)$ & $\begin{array}{l}\text { Standard presence of a varietal, } \\
\text { strawberry, rose }(26.13 \pm 1.36)\end{array}$ & $\begin{array}{l}\text { A little sharp acidity, little lingering } \\
\text { flavour in the mouth, slightly bitter } \\
\text { finish }(33.00 \pm 3.07)\end{array}$ & Superior $(7.38 \pm 0.74)$ & $86.38 \pm 3.93$ \\
\hline
\end{tabular}

Values in the table are the mean values \pm SE.

important roles in winemaking in recent years [9,36,37]. Moreover, according to the results of the preliminary experiment, in the enrichment method, chloramphenicol can prevent the reproduction of bacteria, and alcohol can inhibit the growth of mold, whose colony can cover other colonies. This explained why more yeast colonies were isolated from $\mathrm{CE}$ than from $\mathrm{CnE}$. Yeast isolation from $\mathrm{CnE}$ was disturbed by the huge colonies of mold. Although in this study, no S. cerevisiae was isolated from $\mathrm{CnE}$ or $\mathrm{CE}$, the addition of alcohol could help to isolate $S$. cerevisiae from soil, which had been tried successfully by Sniegowski et al. [38]. Third, a new colony morphology type on the WLN medium was found in this study: the colony morphology of $P$. occidentalis. It is easy to distinguish P. occidentalis colony from other yeast species on the WLN medium with its wrinkled and floury surface. Thus, accumulating different morphology types on the WLN medium make the medium even more valuable in differentiating wine yeasts isolated from the grape must, grape surface [23], vineyard soil, etc.

As shown in Figure 3, three main species of wine yeasts (H. uvarum, $H$. vineae, and S. cerevisiae) dominated the spontaneous fermentation of Cabernet Sauvignon grape must, a common finding in wineries and vineyards around the world [6,37,39]. According to Table 1, P. kluyveri, H. uvarum, S. pombe and T. delbrueckii were frequently emerged in soil samples of different grape varieties. Except for $S$. pombe, the other four yeast species selected from soil samples showed good potential fermenting abilities, and they were also isolated from other wine regions around the world [37,40-42]. But S. pombe can lead to undesirable flavors in wine [43]. Hence, local wineries in Beijing should pay attention to the reproduction of $S$. pombe during fermentation. However, there were still some new discoveries. S. bacillaris (formerly named as Candida zemplinina) usually emerged in the beginning stage of spontaneous fermentation $[9,34,19]$. However, our results showed that S. bacillaris did not appear until the middle stages. These strains existed until the final stages of fermentation, when the ethanol content exceeded $13 \%(\mathrm{v} / \mathrm{v})$, proved that these S. bacillaris strains had high ethanol tolerance. Thus, two S. bacillaris strains M1P9 and M3P9, selected from the 9th day of the fermentation, were selected to be inoculated in subsequent fermentation tests. P. occidentalis (formerly named as Issatchenkia occidentalis), which used to be found in cocoa bean heap fermentation [44] and sourdoughs [45], was seldomly isolated from grapes compared with other non-Saccharomyces. In China, P. occidentalis was also found in Roussanne and Merlot grapes in Huailai, a county near Beijing [32] but was not found in other areas. Thus, it might be a distinctive wine yeast species in Beijing and surrounding areas. Above all, the wine yeast composition at Chateau Lion was relatively simple because the chateau was newly established and has used commercial dry yeast for years. These results emphasize the importance of reserving indigenous wine yeasts, especially in newly established wineries.

According to the dendrogram shown in Figure 4, at least nine different biotypes of $S$. cerevisiae were isolated in the present study. According to the alignment results, the isolated S. cerevisiae strains in Group II and III shared $92.38 \%$ and $97.03 \%$ similarity on the 5.8S ITS sequence, respectively (Figure 4). Especially for commercial strain-R2 and M1D10, presented a similarity of over $99 \%$. Although we did not find strains that were completely identical to commercial wine yeasts, the small differences between sequences of the strains mentioned above may be caused by climate influences, mutation, and so on $[39,46]$. There was also a new research we did recently in Beijing (unpublished); in the Chardonnay grape must during spontaneous fermentation, no Saccharomyces was found. While this 8-year vineyard we studied was far away from wineries, which means there was no commercial wine yeast influence on this relatively isolated vineyard. Some results in another study also showed no Saccharomyces was found in spontaneous fermentation [39]. These results supported that commercial wine yeasts may be the original S. cerevisiae strains in vineyards near wineries. Thus, the high genetic similarity shared by strains in Group II and III indicate that commercial $S$. cerevisiae strains may have already influenced the wine yeast diversity in Chateau Lion in no more than 4 years. Specifically, these strains were isolated from the end of the fermentation (8th to 10th day of the fermentation), when usually the dominant Saccharomyces strains were remaining. Thus, our findings concur with the opinion that the use of only commercial yeasts may lead to the loss of autochthonous wine yeast resources in the wineries $[4,19,47]$. Conflicting opinion was reported by DrumondeNeves et al. [48], which showed human intervention increased the yeast biodiversity, whereas it was based on the yeast biodiversity of grape juice. According to the yeast ecology kinetic during the spontaneous fermentation in this study (Figure 3), it was found that some yeast may be difficult to be isolated from grape juice. From the genomic point of view, the importance of protecting indigenous yeast diversity from being influenced by the commercial yeasts was proven again. Interestingly, it can be concluded that the colony morphology on the WLN medium can reflect some of the genetic relations among strains of the same species (Figure 4). Different clusters in the dendrogram may show different tolerance to alcohol and fermenting ability due to the different stages in which the 
strains were isolated. Hence, further research is needed to integrate the advantages of various indigenous $S$. cerevisiae strains. Except for the current research on the wine fermented by co-cultures of Saccharomyces and non-Saccharomyces [37], a combination of different strains of $S$. cerevisiae can be tried in winemaking. Moreover, exploiting more strains of indigenous wine yeast and applying the strains to wineries is another way to protect the local yeast diversity.

In the inoculated fermentation tests, the two $S$. cerevisiae strains showed good fermentative capabilities. As shown in Figure 5, in the wines inoculated with M1W10 and M2W10, 100\% of the glucose and more than $98 \%$ of the fructose in the initial grape must were used during fermentation. Moreover, the two strains speeded up the fermentation like the commercial yeast F15, requiring only 7 days completing the fermentation. According to the results of sensory tests (Table 2), wines fermented by M1W10 and M2W10 gained higher scores both in aroma, taste and overall quality parts compared with control strain F15. M1W10 and M2W10, two strains in group I, shared low homology on the sequence analysis with the commercial yeasts, indicating that the high possibility that the two strains were indigenous $S$. cerevisiae. Thus, two indigenous $S$. cerevisiae strains with good fermentation performance were selected in this study. However, the two selected S. bacillaris strains showed low fermentation powers, which was in line with previous report [49]. In the wines inoculated with M3P9 and M1P9, only $42.9 \%$ and $41.6 \%$ of the glucose in the initial grape must were transformed after 15 days of fermentation. However, more than $99 \%$ of the fructose was consumed in the two experimental wines. These data indicate that $S$. bacillaris prefers to transform fructose rather than glucose, which leads to the result that fermentation ends with much glucose remaining. In a previous study, similar results were obtained in Minimal Must Medium (a synthetic grape juice medium) [50]. Although S. bacillaris is frequently emerged in China, the specific metabolism of this species in wine has not been reported previously. Compared with the wine inoculated with $S$. cerevisiae, S. bacillaris produced more glycerol, which can create a soft and velvety texture in wine [51]. Besides, fructose is preferentially transformed by S. bacillaris; while glucose is preferentially transformed by $S$. cerevisiae [52]. S. bacillaris can increase the content of glycerol in the wine but will not increase the volatile acidity or decrease the content of succinic acid and lactic acid. In addition, S. bacillaris can help to take full advantage of the fructose, avoiding the deterioration in wine caused by residual fructose. Although the wines made with M3P9 and M1P9 did not gain high scores in sensory tests, a combination of $S$. bacillaris and $S$. cerevisiae deserves to be studied as co-culture starters in wine making in the future.

In summary, through this preliminary study, we can find that there are abundant biodiversity of indigenous wine yeasts in Beijing. The new colony morphology of $P$. occidentalis on the WLN medium was reported first time. Two indigenous $S$. cerevisiae selected from grape must of Chateau Lion (Beijing), especially M2W10, were evaluated and found to be the yeast with good fermentation performances. We recommended $S$. bacillaris, a popular wine yeast species in China, can be studied as a co-culture starter species with $S$. cerevisiae in the future, since $S$. bacillaris can increase the content of glycerol and transform more fructose than $S$. cerevisiae during fermentation. Moreover, our results show that there is an urgent need to exploit indigenous wine yeast in wineries in Beijing, especially for those wineries who want to make unusual wines.

\section{CONFLICTS OF INTEREST}

The authors declare that the research was conducted in the absence of any commercial or financial relationships that could be construed as a potential conflicts of interest.

\section{AUTHORS' CONTRIBUTION}

QZ, JZ and WH designed experiments. QZ carried out experiments. QZ and HL analyzed experimental results. HL gave essential technical guidance. QZ and XS wrote the manuscript.

\section{ACKNOWLEDGMENTS}

The authors express their gratitude for funding from National Key R\&D Program of China (2016YFD0400500), China Spark Program (2015GA600003) and fund from Chateau Lion. We also express our gratitude to Dongxue Liu, Song Shi, Jun Cai, Menglong Hu, Yang $\mathrm{Fu}$, and Jingkun $\mathrm{Ou}$ for their valuable technical assistance.

\section{REFERENCES}

[1] OIV. State of the vitiviniculture world market: state of the sector in 2018 [R/OL]. 2019. Available from: http://www.oiv.int/ public/medias/6679/en-oiv-state-of-the-vitiviniculture-worldmarket-2019.pdf.

[2] Comitini F, Capece A, Ciani M, Romano P. New insights on the use of wine yeasts. Curr Opin Food Sci 2017;13:44-9.

[3] Fleet GH. Yeast interactions and wine flavour. Int J Food Microbiol 2003;86:11-22.

[4] Suárez-Lepe JA, Morata A. New trends in yeast selection for winemaking. Trends Food Sci Technol 2012;23:39-50.

[5] Nykänen L. Formation and occurrence of flavor compounds in wine and distilled alcoholic beverages. Am J Enol Vitic 1986;37:84-96.

[6] Steensels J, Verstrepen KJ. Taming wild yeast: potential of conventional and nonconventional yeasts in industrial fermentations. Annu Rev Microbiol 2014;68:61-80.

[7] Romano P, Fiore C, Paraggio M, Caruso M, Capece A. Function of yeast species and strains in wine flavour. Int J Food Microbiol 2003;86:169-80.

[8] Garofalo C, Berbegal C, Grieco F, Tufariello M, Spano G, Capozzi V. Selection of indigenous yeast strains for the production of sparkling wines from native Apulian grape varieties. Int J Food Microbiol 2018;285:7-17.

[9] Lin MMH, Boss PK, Walker ME, Sumby KM, Grbin PR, Jiranek V. Evaluation of indigenous non-Saccharomyces yeasts isolated from a South Australian vineyard for their potential as wine starter cultures. Int J Food Microbiol 2020;312:108373.

[10] Ortiz MJ, Barrajón N, Baffi MA, Arévalo-Villena M, Briones A. Spontaneous must fermentation: identification and biotechnological properties of wine yeasts. LWT - Food Sci Technol 2013;50:371-7. 
[11] Francesca N, Sannino C, Settanni L, Corona O, Barone E, Moschetti G. Microbiological and chemical monitoring of Marsala base wine obtained by spontaneous fermentation during large-scale production. Ann Microbiol 2014;64:1643-57.

[12] Green SR, Gray PP. A differential procedure applicable to bacteriological investigation in brewing. Proc Annual Meeting Am Soc Brew Chem 1950;8:19-32.

[13] Pallmann CL, Brown JA, Olineka TL, Cocolin L, Mills DA, Bisson LF. Use of WL medium to profile native flora fermentations. Am J Enol Vitic 2001;52:198-203.

[14] Marsit S, Dequin S. Diversity and adaptive evolution of Saccharomyces wine yeast: a review. FEMS Yeast Res 2015;15:fov067.

[15] Torija MJ, Rozès N, Poblet M, Guillamón JM, Mas A. Yeast population dynamics in spontaneous fermentations: comparison between two different wine-producing areas over a period of three years. Antonie van Leeuwenhoek 2001;79:345-52.

[16] Tofalo R, Perpetuini G, Fasoli G, Schirone M, Corsetti A, Suzzi G. Biodiversity study of wine yeasts belonging to the "terroir" of Montepulciano d'Abruzzo "Colline Teramane" revealed Saccharomyces cerevisiae strains exhibiting atypical and unique 5.8S-ITS restriction patterns. Food Microbiol 2014;39:7-12.

[17] Csernus O, Pomázi A, Magyar I. Isolation, characterisation, and selection of wine yeast strains in Etyek-Buda wine district, Hungary. Acta Alimentaria 2014;43:489-500.

[18] Stockert CM, Bisson LF, Adams DO, Smart DR. Nitrogen status and fermentation dynamics for merlot on two rootstocks. Am J Enol Vitic 2013;64:195-202.

[19] Sun Y, Guo J, Liu F, Liu Y. Identification of indigenous yeast flora isolated from the five winegrape varieties harvested in Xiangning, China. Antonie van Leeuwenhoek 2014;105:533-40.

[20] Wang C, Liu Y. Dynamic study of yeast species and Saccharomyces cerevisiae strains during the spontaneous fermentations of Muscat blanc in Jingyang, China. Food Microbiol 2013;33:172-7.

[21] Di Maro E, Ercolini D, Coppola S. Yeast dynamics during spontaneous wine fermentation of the Catalanesca grape. Int J Food Microbiol 2007;117:201-10.

[22] Domizio P, Lencioni L, Ciani M, Di Blasi S, Pontremolesi C, Sabatelli MP. Spontaneous and inoculated yeast populations dynamics and their effect on organoleptic characters of Vinsanto wine under different process conditions. Int J Food Microbiol 2007;115:281-9.

[23] Martini A, Ciani M, Scorzetti G. Direct enumeration and isolation of wine yeasts from grape surfaces. Am J Enol Vitic 1996;47:435-40.

[24] Mortimer R, Polsinelli M. On the origins of wine yeast. Res Microbiol 1999;150:199-204.

[25] Liu X, Jia B, Sun X, Ai J, Wang L, Wang C, et al. Effect of initial $\mathrm{pH}$ on growth characteristics and fermentation properties of Saccharomyces cerevisiae. J Food Sci 2015;80:M800-M8.

[26] Cavazza A, Grando MS, Zini C. Rilevazione della flora microbica di mosti e vini. Vignevini 1992;9:17-20.

[27] Guillamón JM, Sabaté J, Barrio E, Cano J, Querol A. Rapid identification of wine yeast species based on RFLP analysis of the ribosomal internal transcribed spacer (ITS) region. Arch Microbiol 1998;169:387-92.

[28] Tamura K, Stecher G, Peterson D, Filipski A, Kumar S. MEGA6: molecular evolutionary genetics analysis version 6.0. Mol Biol Evol 2013;30:2725-9.
[29] GB/T 15038-2006. Analytical methods of wine and fruit wine. Beijing: The National Standard of China; 2006.

[30] Ronald SJ. Wine tasting. 2nd ed. Burlington: Elsevier Inc.; 2009.

[31] Takahashi M, Ohta T, Masaki K, Mizuno A, Goto-Yamamoto N. Evaluation of microbial diversity in sulfite-added and sulfite-free wine by culture-dependent and -independent methods. J Biosci Bioeng 2014;117:569-75.

[32] Sun H, Ma H, Hao M, Pretorius IS, Chen S. Identification of yeast population dynamics of spontaneous fermentation in Beijing wine region, China. Ann Microbiol 2009;59:69-76.

[33] Li SS, Cheng C, Li Z, Chen JY, Yan B, Han BZ, et al. Yeast species associated with wine grapes in China. Int J Food Microbiol 2010;138:85-90.

[34] Li E, Liu A, Xue B, Liu Y. Yeast species associated with spontaneous wine fermentation of Cabernet Sauvignon from Ningxia, China. World J Microbiol Biotechnol 2011;27:2475-82.

[35] Jolly NP, Varela C, Pretorius IS. Not your ordinary yeast: nonSaccharomyces yeasts in wine production uncovered. FEMS Yeast Res 2014;14:215-37.

[36] Nisiotou A, Mallouchos A, Tassou C, Banilas G. Indigenous yeast interactions in dual-starter fermentations may improve the varietal expression of moschofilero wine. Front Microbiol 2019;10:1712.

[37] Liu PT, Lu L, Duan CQ, Yan GL. The contribution of indigenous non-Saccharomyces wine yeast to improved aromatic quality of Cabernet Sauvignon wines by spontaneous fermentation. LWT Food Sci Technol 2016;71:356-63.

[38] Sniegowski PD, Dombrowski PG, Fingerman E. Saccharomyces cerevisiae and Saccharomyces paradoxus coexist in a natural woodland site in North America and display different levels of reproductive isolation from European conspecifics. FEMS Yeast Res 2002;1:299-306.

[39] Drumonde-Neves J, Franco-Duarte R, Lima T, Schuller D, Pais C. Association between grape yeast communities and the vineyard ecosystems. PLoS One 2017;12:e0169883.

[40] Wang C, Mas A, Esteve-Zarzoso B. Interaction between Hanseniaspora uvarum and Saccharomyces cerevisiae during alcoholic fermentation. Int J Food Microbiol 2015;206:67-74.

[41] Contreras A, Curtin C, Varela C. Yeast population dynamics reveal a potential 'collaboration' between Metschnikowia pulcherrima and Saccharomyces uvarum for the production of reduced alcohol wines during Shiraz fermentation. Appl Microbiol Biotechnol 2015;99:1885-95.

[42] Ciani M, Comitini F, Mannazzu I, Domizio P. Controlled mixed culture fermentation: a new perspective on the use of non-Saccharomyces yeasts in winemaking. FEMS Yeast Res 2010;10:123-33.

[43] Kenneth CF, Charles GE. Wine Microbiology. New York City (NYC): Springer Science Business Media, LLC; 2007.

[44] Daniel HM, Vrancken G, Takrama JF, Camu N, De Vos P, De Vuyst L. Yeast diversity of Ghanaian cocoa bean heap fermentations. FEMS Yeast Res 2009;9:774-83.

[45] Nuobariene L, Hansen ÅS, Arneborg N. Isolation and identification of phytase-active yeasts from sourdoughs. LWT - Food Sci Technol 2012;48:190-6.

[46] Valero E, Cambon B, Schuller D, Casal M, Dequin S. Biodiversity of Saccharomyces yeast strains from grape berries of wineproducing areas using starter commercial yeasts. FEMS Yeast Res 2007;7:317-29. 
[47] Blanco P, Ramilo A, Cerdeira M, Orriols I. Genetic diversity of wine Saccharomyces cerevisiae strains in an experimental winery from Galicia (NW Spain). Antonie van Leeuwenhoek 2006;89:351-7.

[48] Drumonde-Neves J, Franco-Duarte R, Lima T, Schuller D, Pais C. Yeast biodiversity in vineyard environments is increased by human intervention. PLoS One 2016;11:e0160579.

[49] Comitini F, Gobbi M, Domizio P, Romani C, Lencioni L, Mannazzu I, et al. Selected non-Saccharomyces wine yeasts in controlled multistarter fermentations with Saccharomyces cerevisiae. Food Microbiol 2011;28:873-82.
[50] Domizio P, Liu Y, Bisson LF, Barile D. Use of non-Saccharomyces wine yeasts as novel sources of mannoproteins in wine. Food Microbiol 2014;43:5-15.

[51] Moreno-Arribas MV, Polo MC. Wine Chemistry and Biochemistry. New York City (NYC): Springer Science Business Media, LLC; 2009.

[52] Zinnai A, Venturi F, Sanmartin C, Quartacci MF, Andrich G. Kinetics of D-glucose and D-fructose conversion during the alcoholic fermentation promoted by Saccharomyces cerevisiae. J Biosci Bioeng 2013;115:43-9. 